\title{
Linking biodiversity and Global Environmental Changes in Brazilian coastal habitats
}

The Earth's climate is changing at a time when society is re-evaluating its relationship with nature and with the services that natural systems provide to human societies. Human actions, which are the major cause of these changes, also reduce the ability of ecological systems to cope with and adapt to the new scenarios. As a result, in the near future only the biota but humanity as a whole will feel the effects of our unsustainable way of life. Among the expected effects, there is the compromising of ecosystem services that are the basis of life and the human economy in diferente parts of the globe.

Oceans are central to the climate system, recycling half of the oxygen that we breathe and absorbing half of the carbon dioxide that we emit through the burning of fossil fuels. The oceans hold $97 \%$ of the Earth's water and $95 \%$ of all mobile carbon, providing food and livelihoods. Determining the effects of Global Environmental Changes (GECs) on the oceans are critical to understanding what is changing, how is it changing, and how these changes will influence society. Direct and indirect effects of GECs on the marine environment are already perceptible, but others can only be projected based on observations, experimentation and modeling efforts. We have only a rudimentary understanding of the sensitivity, vulnerability and adaptability of natural and managed marine ecosystems to GECs, especially in the South Atlantic.

On the Brazilian coast, the existing baseline, monitoring and predictive studies are insufficient to understand the detrimental effects of GECs. The lack of long-term studies of biodiversity has left Brazil far behind in global assessments of the consequences of GECs on coastal ecosystems. In contrast, the Brazilian coastline has vegetated ecosystems (mangroves, salt marshes, and seagrass and rhodolith beds) that together contain hundreds of millions of tons of stored carbon, making Brazil a good place to test new mechanisms to evaluate and conserve blue carbon. To promote the development of a regional science-policy agenda to respond to the urgent demand for sound scientific advice in the face of rapid changes to marine coastal ecosystems, it is necessary to integrate baseline studies to assess the habitat distribution and quality of the ecosystems, as well as the human threats and risks associated with local and regional climate-change scenarios; promote strategic monitoring of physical and biological parameters to fill critical gaps in knowledge; and provide an early warning system of GECs to coastal communities. There is also a need to refine regional and local scenarios of threats related to GECs, to assess the uncertainties, risks and thresholds at the organism and ecosystem levels.

It is imperative to integrate Brazilian researchers and institutions in order to promote the consolidation of existing knowledge and the implementation of a broad and continuous observational network. The Network for Monitoring Benthic Coastal Habitats (ReBentos) was created to detect the effects of regional and global environmental changes on benthic habitats, by creating a time-series of data on biodiversity along the Brazilian coast. ReBentos is linked to the Coastal Zones Branch of the Climate Network, hosted by the Ministry of Science, Technology and Innovation, and the National Institute of Science and Technology for Climate Change. The network was supported in its early phase by the National Counsel of Technological and Scientific Development (CNPq), São Paulo Research Foundation (FAPESP), and Coordination for the Improvement of Higher-Education Personnel (CAPES). Currently, ReBentos (http://rebentos.org/) comprises 166 active researchers along the entire Brazilian coast, belonging to 57 educational/research institutions, both national and international, and 17 coastal states.

The strategy adopted by ReBentos was to define standardized methods for biodiversity sampling, processing and data analysis, and for measuring abiotic and anthropogenic factors. The free-access e-book Protocolos para o Monitoramento de Habitats Bentônicos Costeiros (in English: Protocols for Monitoring Benthic Coastal Habitats; http://books.scielo.org/id/ $\mathrm{x} 49 \mathrm{kz}$ ) recommends simple, rapid and low-cost methods for continuous and long-term monitoring in the diferente benthic habitats along the Brazilian coast. Based on this monitoring effort, an inventory will be assembled to increase knowledge of marine biodiversity along the Brazilian coast, and eventually to allow understanding of possible changes in the biota due to natural and/or anthropogenic events, within the context of GECs.

As a parallel strategy, ReBentos realized the need to develop a broad understanding of current knowledge on the coastal marine benthic biodiversity in different habitats along the Brazilian coast, as well as their particular susceptibilities to potential impacts of GECs. The synthesis of knowledge presented in this special issue emerges from this need, with nine articles covering different habitats including estuaries, the macrofauna and meiofauna of sandy beaches, rocky shores, coral reefs, mangroves and salt marshes, rhodolith and seagrass beds and education. 
These reviews followed general guidance from the ReBentos coordinators regarding the logical structure, with a description of the habitats including their characterization, distribution along the coast, ecosystem services, and potential responses to local and global threats. They also integrate existing knowledge regarding biodiversity studies and their historical evolution, location, spatial and thematic gaps, methods employed, existence of time series, and approaches related to the evaluation of the effect of GECs.

As ReBentos also has an environmental-education strategy aiming to develop a discussion on the issue of GECs on marine and coastal ecosystems, in order to foster and encourage changes in attitudes and values in relation to these environments and their biodiversity, a review of the approaches and initiatives undertaken along the Brazilian coast was also prepared and incorporated into this volume.

The preparation of this special issue on the synthesis of knowledge of coastal marine biodiversity and the effects of GECs on the Brazilian coast constitutes an important milestone for coastal benthic research in Brazil, providing a broad overview of the current state of knowledge. The gaps identified in both the geographical and thematic aspects influenced the selection of the sites that are being monitored by ReBentos, as well as environmental education initiatives. We believe that this special issue will play an important role in supporting future studies on biodiversity and GECs in Brazilian coastal habitats.

\author{
Alexander Turra ${ }^{1}$ \\ Márcia Regina Denadai ${ }^{1}$ \\ General Coordinators, ReBentos
}

\footnotetext{
${ }^{1}$ Universidade de São Paulo Instituto Oceanográfico

(Praça do Oceanográfico, 191 - Cidade Universitária, 05508-120 - São Paulo - SP)
} 